\title{
A SUPERIOR KIRCHHOFF METHOD FOR AEROACOUSTIC NOISE PREDICTION: The Ffowcs Williams-Hawkings equation
}

\author{
Kenneth S. Brentner \\ NASA Langley Research Center
}

Presented at the 134th Meeting of the Acoustical Society of America, San Diego, CA, December 1-5, 1997

NASA Lanqlev Research Center. Hampton. VA 


\section{Introduction}

- Prediction of aeroacoustic noise important

$>$ all new aircraft must meet noise certification requirements

> local noise standards can be even more stringent

> NASA noise reduction goal: reduce perceived noise levels by a factor of two in 10 years

- Several prediction methods available

> direct computation

- CFD based methods

- near field only

- best coupled with integral method for far-field prediction

> Acoustic Analogy (Ffowcs Williams-Hawkings Equation)

> Kirchhoff formula

- Confusion over relationship between methods exists

\section{NASA Lanqlev Research Center. Hampton. VA}




\section{Comments on Integral Methods}

- Technology

$>$ acoustic formulations and algorithms mature

- widely used for rotating blade noise prediction

- potentially useful for airframe noise, engine noise, etc.

$>$ flow field computation feasible in many cases

- required for input data

- provided by CFD

$>$ high quality experiments aid validation

- This talk will demonstrate the superiority of the FW-H approach over the Kirchhoff method for aeroacoustics

$>$ analytically

$>$ numerically

\section{NASA Lanqlev Research Center. Hampton, VA}




\section{Advantages and Disadvantages}

- FW-H method

+ three source terms (thickness, loading, quadrupole) have physical meaning

+ three source terms are independent

+ mature and robust algorithms

- quadrupole source is a volume source (more computational resources needed when volume integration included)

- Kirchhoff method

+ surface sources (only surface integration required)

+ applicable to problems described by the wave equation

- source terms not easily related to flow physics or design parameters

- not as much experience with algorithms for Kirchhoff problems

- Analytical/Numerical comparison needed

\section{NASA Lanqlev Research Center. Hampton. VA}




\section{Analytical Comparison: FW-H Derivation Procedure}

- Embed exterior flow problem in unbounded space

$>$ define generalized functions valid throughout entire space

$>$ interpret derivatives as generalized differentiation

$$
\begin{aligned}
\tilde{\rho} & =\left\{\begin{array}{cc}
\rho & f>0 \\
\rho_{o} & f<0
\end{array}\right. \\
\rho \tilde{u}_{i} & =\left\{\begin{array}{cc}
\rho u_{i} & f>0 \\
0 & f<0
\end{array}\right. \\
\tilde{P}_{i j} & =\left\{\begin{array}{cc}
P_{i j} & f>0 \\
0 & f<0
\end{array}\right.
\end{aligned}
$$

- Generalized conservation equations:

$$
\begin{array}{cc}
\frac{\bar{\partial} \tilde{\rho}}{\partial t}+\frac{\bar{\partial} \rho \tilde{u}_{i}}{\partial x_{i}}=\left(\rho^{\prime} \frac{\overline{\partial f}}{\partial t}+\rho u_{i} \frac{\bar{\partial} f}{\partial x_{i}}\right) \delta(f) & \text { continuity } \\
\frac{\bar{\partial} \rho \tilde{u}_{i}}{\partial t}+\frac{\bar{\partial} \rho \tilde{u}_{i} \tilde{u}_{j}}{\partial x_{j}}+\frac{\bar{\partial} \tilde{P}_{i j}}{\partial x_{j}}=\left(\rho u_{i} \frac{\partial f}{\partial t}+\left(\rho u_{i} u_{j}+P_{i j}\right) \frac{\partial f}{\partial x_{i}}\right) \delta(f) & \text { momentum }
\end{array}
$$




\section{Analytical Comparison: FW-H Derivation Procedure}

- Manipulate conservation laws into form of inhomogeneous wave equation

$$
\begin{array}{ll}
\square^{2} p^{\prime}(\vec{x}, t)=\frac{\bar{\partial}^{2}}{\partial x_{i} \partial x_{j}}\left[T_{i j} H(f)\right] & \frac{\partial f}{\partial t}=-v_{n} \\
-\frac{\partial}{\partial x_{i}}\left[\left(P_{i j} \hat{n}_{j}+\rho u_{i}\left(\left(u_{n}-v_{n}\right)\right) \delta(f)\right]\right. & \frac{\partial f}{\partial x_{i}}=\hat{n}_{i} \\
+\frac{\partial}{\partial t}\left[\left(\rho_{o} v_{n}+\rho\left(u_{n}-v_{n}\right) \delta(f)\right]\right. &
\end{array}
$$

- Don't assume integration surface $f=0$ is coincident with body

$>$ given in this form by Ffowes Williams

$>$ demonstrated for rotors by di Francescantonio; Brentner \& Farassat

\section{NASA Lanqlev Research Center. Hampton. VA}




\section{Analytical Comparison: Kirchhoff Derivation Procedure}

- Use embedding procedure on wave equation

$>$ define generalized pressure perturbation:

$$
\tilde{p}^{\prime}= \begin{cases}p^{\prime} & f>0 \\ 0 & f<0\end{cases}
$$

$>$ use generalized derivatives

> generalized wave equation is Kirchhoff governing equation:

$$
\begin{aligned}
\square^{2} p^{\prime}(\vec{x}, t) & =-\left(\frac{\partial p^{\prime}}{\partial t} \frac{M_{n}}{c}+\frac{\partial p^{\prime}}{\partial n}\right) \delta(f)-\frac{\partial}{\partial t}\left(p^{\prime} \frac{M_{n}}{c} \delta(f)\right)-\frac{\partial}{\partial x_{i}}\left(p^{\prime} \hat{n}_{i} \delta(f)\right) \\
& \equiv Q_{k i r}
\end{aligned}
$$

NASA Lanqlev Research Center. Hampton. VA 


\section{Source Term Comparison}

- Manipulate FW-H source terms into form of Kirchhoff source terms (inviscid fluid)

$$
\begin{aligned}
\square^{2} p^{\prime}(\vec{x}, t)= & Q_{k i r}+\frac{\bar{\partial}^{2}}{\partial x_{i} \partial x_{j}}\left[T_{i j} H(f)\right] \\
& -\frac{\partial}{\partial x_{j}}\left[\rho u_{i} u_{j}\right] \hat{n}_{i} \delta(f)-\frac{\partial}{\partial x_{j}}\left[\rho u_{i} u_{n} \delta(f)\right] \\
& +\frac{\partial}{\partial t}\left[p^{\prime}-c^{2} \rho^{\prime}\right] \frac{M_{n}}{c} \delta(f)+\frac{\partial}{\partial t}\left[\left(p^{\prime}-c^{2} \rho^{\prime}\right) \frac{M_{n}}{c} \delta(f)\right]
\end{aligned}
$$

- Extra source terms are 2nd order in perturbations quantities

- FW-H and Kirchhoff source terms

$>$ equivalent in linear region $\quad\left(p^{\prime} \approx c^{2} \rho^{\prime} \quad u_{i}<<1\right)$

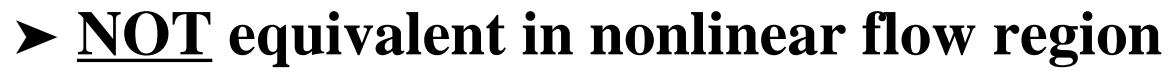

NASA Lanqlev Research Center. Hampton. VA 


\section{Integral Formulation of FW-H equation}

- New variables put FW-H equation into standard form

$$
Q=\rho u_{n}-\rho^{\prime} v_{n} ; \quad L_{i}=P_{i j}+\rho u_{i}\left(u_{n}-v_{n}\right)
$$

hence

$$
\square^{2} p^{\prime}(\vec{x}, t)=\frac{\bar{\partial}^{2}}{\partial x_{i} \partial x_{j}}\left[T_{i j} H(f)\right]-\frac{\partial}{\partial x_{i}}\left[L_{i} \delta(f)\right]+\frac{\partial}{\partial t}[Q \delta(f)]
$$

- Integral representation of solution (formulation 1A)

$$
\begin{aligned}
4 \pi p^{\prime}(\vec{x}, t) & =\int_{f=0}\left[\frac{\dot{Q}+\dot{L}_{r} / c}{r\left(1-M_{r}\right)^{2}}\right]_{r e t} d S+\int_{f=0}\left[\frac{L_{r}-L_{M}}{r^{2}\left(1-M_{r}\right)^{2}}\right]_{r e t} d S \\
& +\int_{f=0}\left[\frac{\left(Q+L_{r} / c\right)\left(r \dot{M}_{r}+c\left(M_{r}-M^{2}\right)\right)}{r^{2}\left(1-M_{r}\right)^{3}}\right]_{r e t} d S
\end{aligned}
$$

NASA Lanqlev Research Center. Hampton, VA 


\section{Kirchhoff Formulation for Moving surfaces}

- Kirchhoff integral formulation

$$
\begin{aligned}
& 4 \pi p^{\prime}(\vec{x}, t)=\int_{f=0}\left[\frac{E_{1}}{r\left(1-M_{r}\right)}\right]_{r e t} d S+\int_{f=0}\left[\frac{p^{\prime} E_{2}}{r^{2}\left(1-M_{r}\right)}\right]_{r e t} d S \\
& \text { where }
\end{aligned}
$$

$$
\begin{aligned}
& E_{1}=\left(M_{n}^{2}-1\right) \frac{\partial p^{\prime}}{\partial n}+M_{n} \vec{M}_{t} \cdot \nabla_{2} p^{\prime}-\frac{M_{n}}{c} \dot{p}^{\prime} \\
& +\frac{1}{c\left(1-M_{r}\right)}\left[\left(\dot{n}_{r}-\dot{M}_{r}-\dot{n}_{M}\right) p^{\prime}+\left(\cos \theta-M_{n}\right) \dot{p}^{\prime}\right]+\frac{1}{c\left(1-M_{r}\right)^{2}}\left[\dot{M}_{r}\left(\cos \theta-M_{n}\right) p^{\prime}\right] \\
& E_{2}=\frac{\left(1-M^{2}\right)}{\left(1-M_{r}\right)^{2}}\left(\cos \theta-M_{n}\right)
\end{aligned}
$$

NASA Lanqlev Research Center. Hampton, VA 


\section{Numerical Comparison}

- Kirchhoff code (RKIR)

> numerical implementation of Kirchhoff integration

> code developed for helicopter rotors (Purdue/Sikorsky/NASA LaRC)

- Prototype code developed (FW-H/RKIR)

> based on RKIR (Rotating Kirchhoff code - rotor noise prediction)

$>$ utilizes Farassat's formulation 1A

> quadrupole source neglected; could be included

- Cases for comparison

$>$ hovering rotor

$>$ rotor in forward flight

> viscous flow over a circular cylinder

NASA Lanqlev Research Center. Hampton. VA 


\section{Numerical Comparison: UH-1H hovering rotor}

- UH-1H rotor

> 1/7th scale model

$>$ untwisted blade

- Test setup (Purcell)

> Hover, $\mathrm{M}_{\mathrm{H}}=\mathbf{0 . 8 8}$

> inplane microphone, 3.09 R from hub

- Flow-field computation

$>$ full potential flow solver used (FPRBVI)

$>80 \times 36 \times 24$ grid (somewhat coarse)

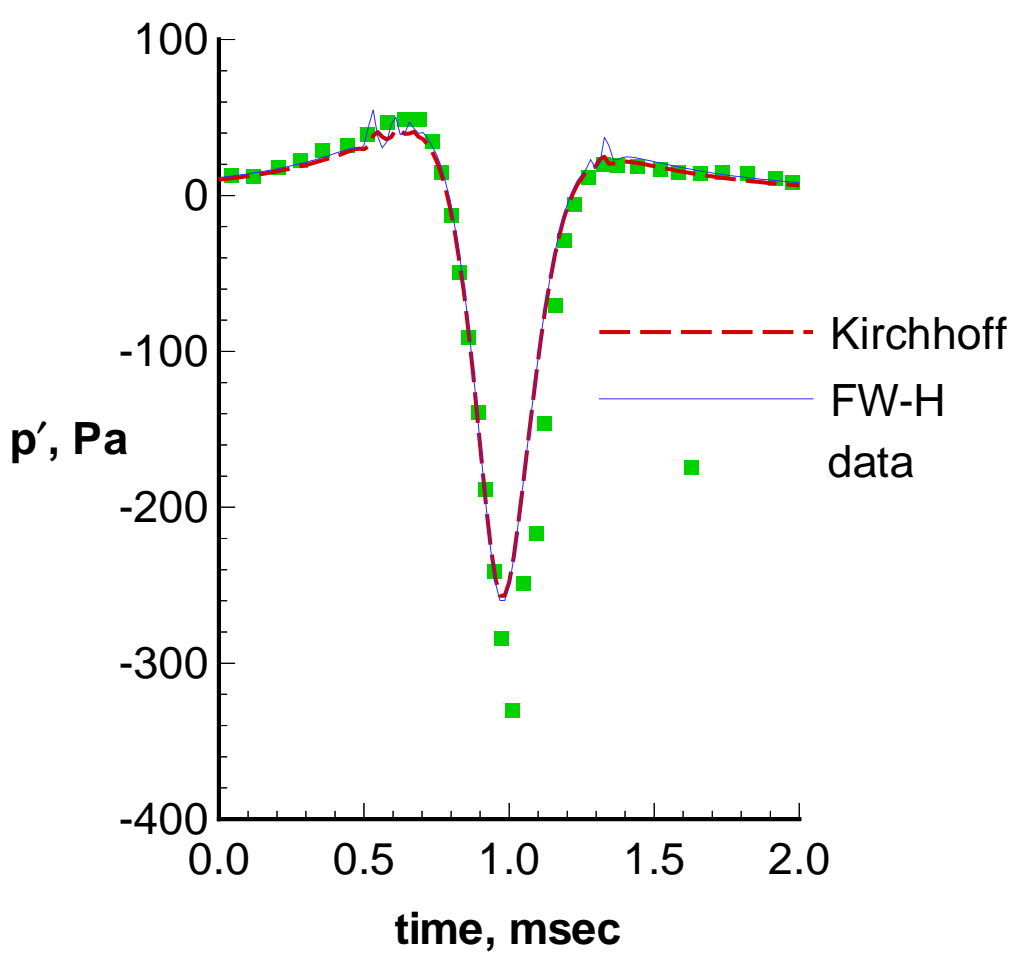

NASA Lanqlev Research Center. Hampton. VA 


\section{Numerical Comparison: Sensitivity to Surface Placement}

- A principal advantage of the $\mathrm{FW}-\mathrm{H}$ approach is insensitivity to surface placement

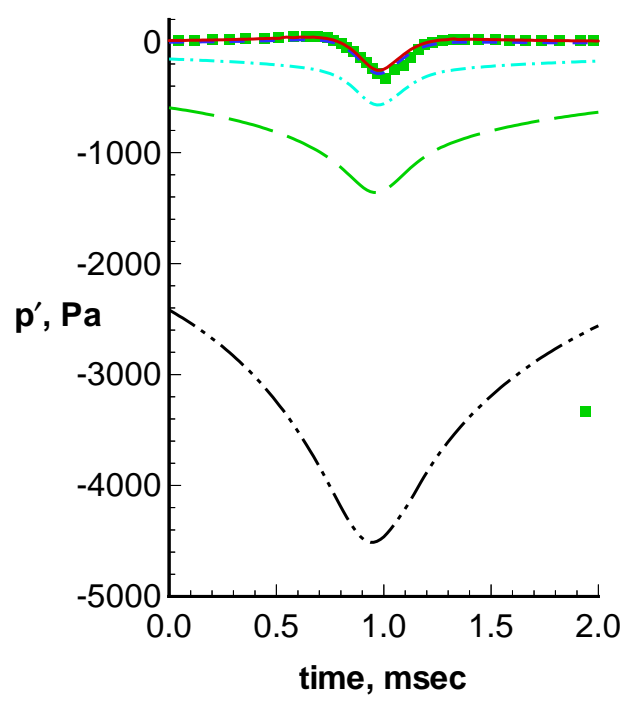

Kirchhoff
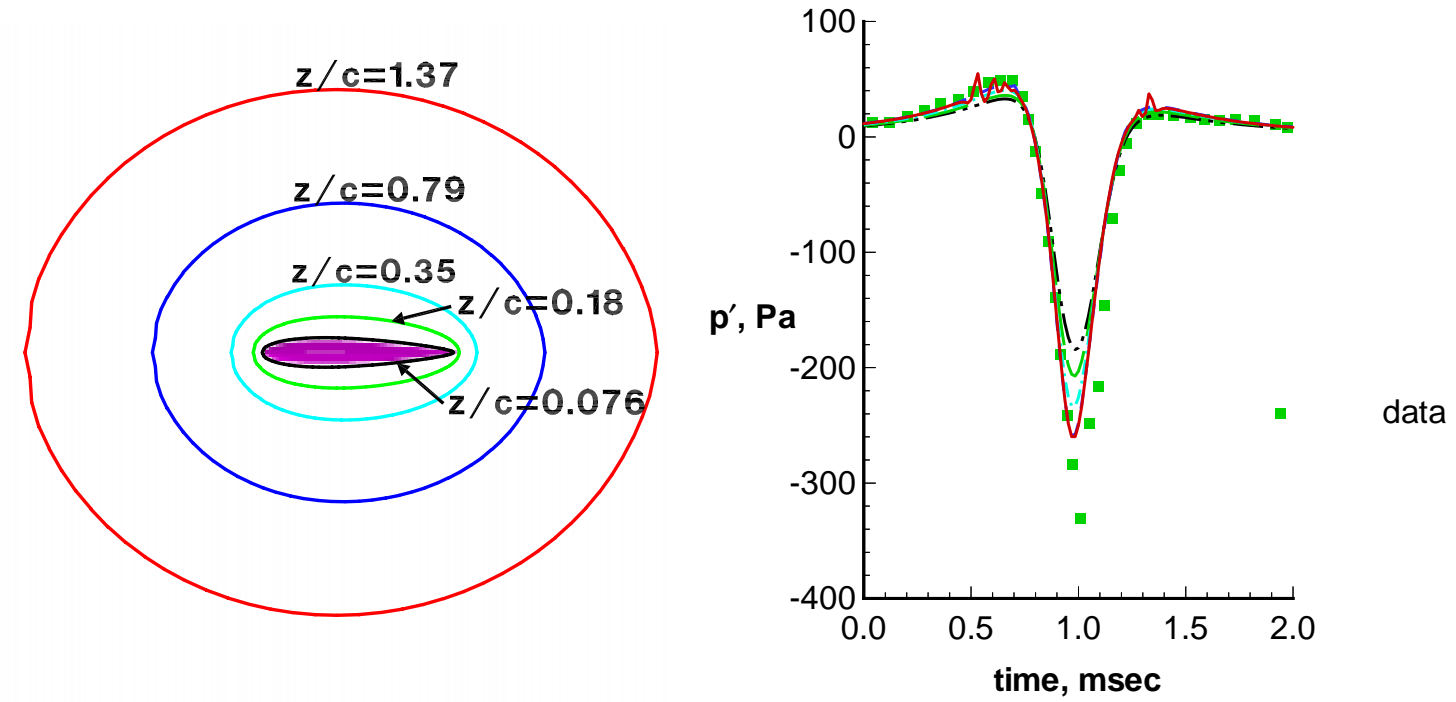

$$
\text { FW-H }
$$

(Note difference in pressure scales)

NASA Langlev Research Center. Hampton. VA 


\section{Identification of Noise Components}

- Compare components from off FW-H/RKIR with WOPWOP+

$>\mathrm{UH}-1 \mathrm{H}$ rotor in hover

> Hover solution from TURNS (Baeder)

- Two predictions necessary with FW-H/RKIR

$>$ thickness and loading from surface coincident with rotor blade

> total signal from a surface approximately 1.5 chords away from blade.

- New application of FW-H equation retains advantage of

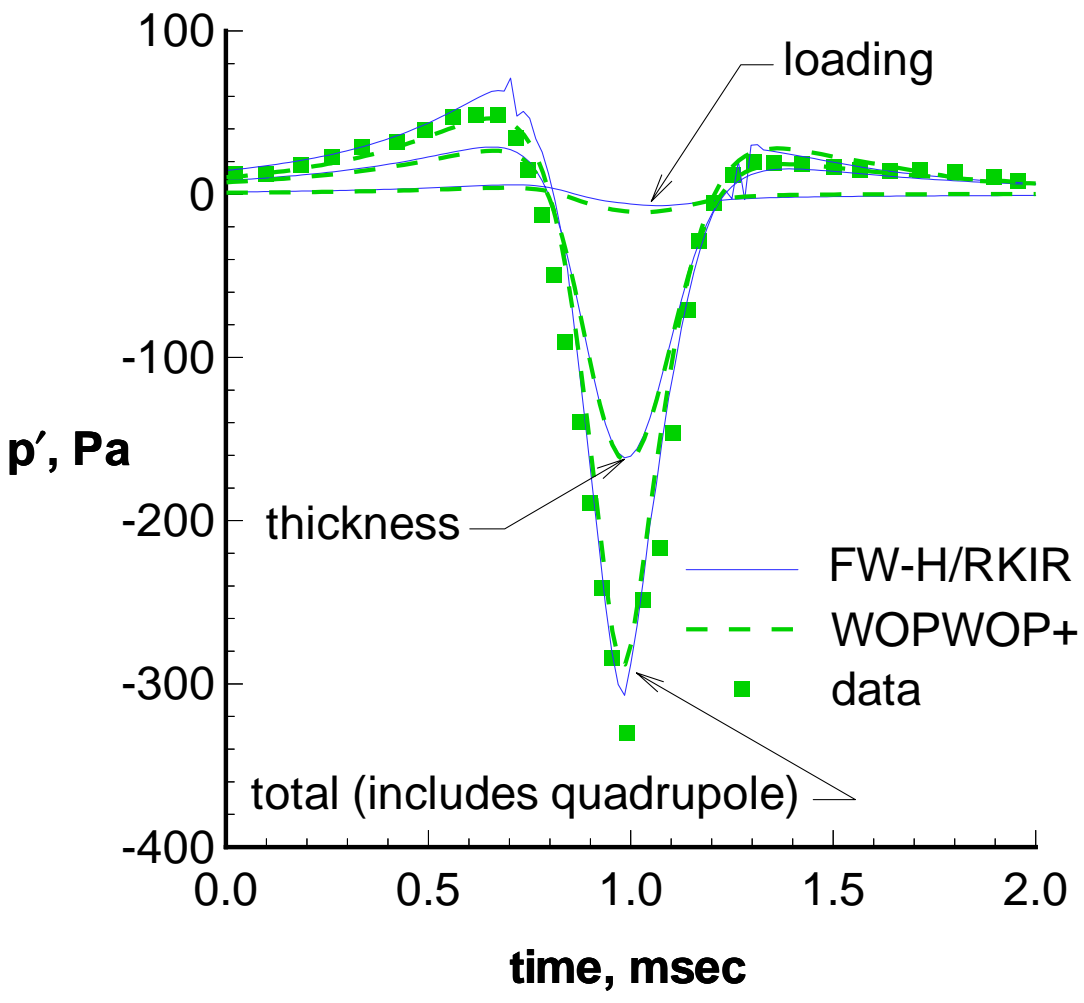
predicting noise components

NASA Lanqlev Research Center. Hampton, VA 


\section{Numerical Comparison: Forward Flight Case}

- Test setup (Schmitz et al.)

$>$ Operational Loads Survey (OLS) 1/7 scale model rotor

$>3$ inplane microphone used for comparison

- Operating conditions

$>\mathrm{M}_{\mathrm{AT}}=0.84$

$>\mu=0.27$

- Flow-field computation

> flow solver: full potential code for rotors (FPRBVI)

$>80 \times 36 \times 24$ grid

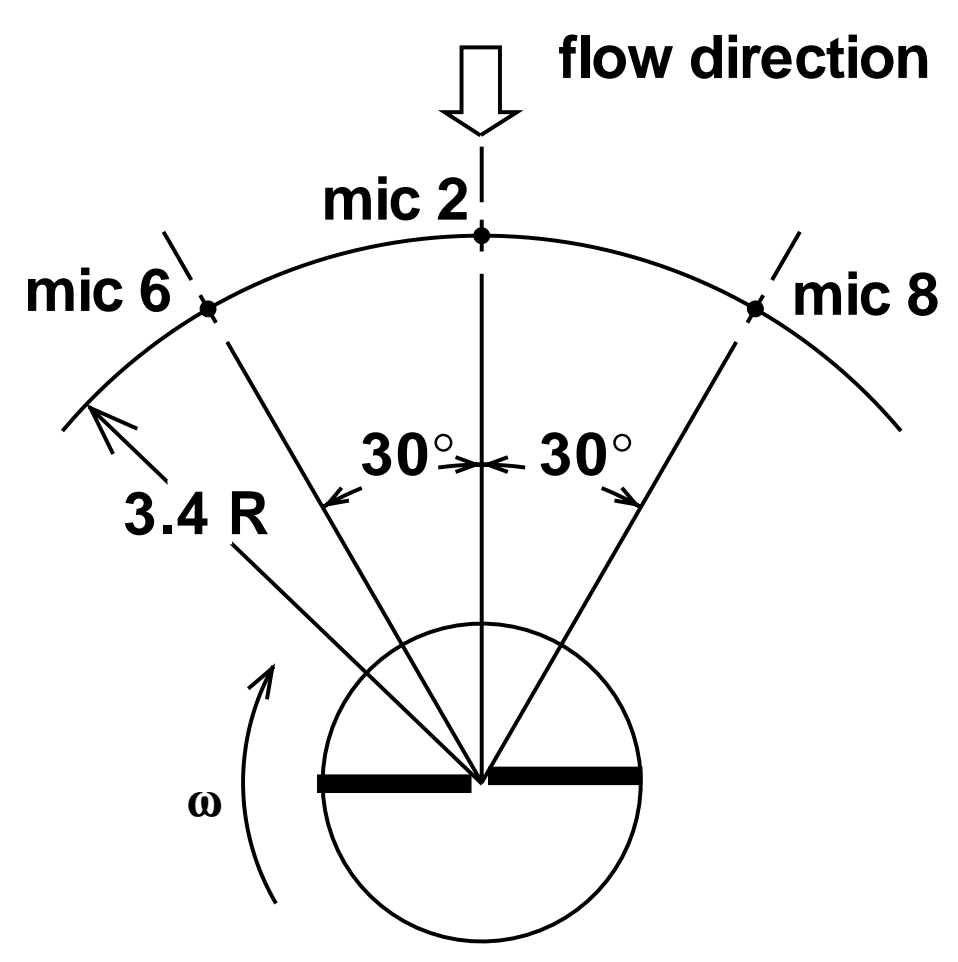

NASA Lanqlev Research Center. Hampton, VA 


\section{Numerical Comparison: Forward Flight Case}

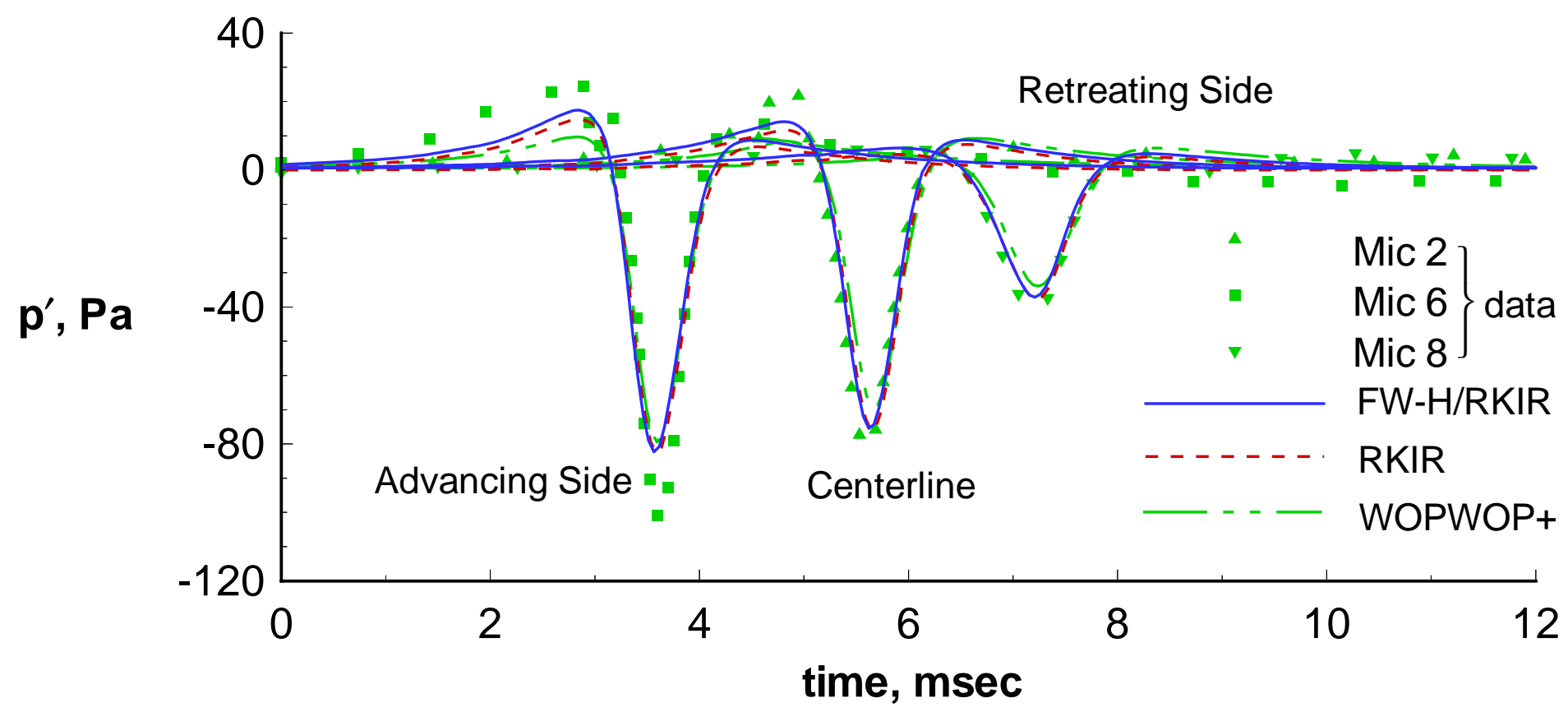

- Advancing-side acoustic pressure underpredicted

- Agreement with data is good
- All three codes agree with each other

NASA Lanqlev Research Center, Hampton. VA 


\section{Numerical Comparison: Circular Cylinder Flow}

- Problem:

> Viscous flow over a circular cylinder

2D, unsteady laminar CFD computation, $R e=1000$.

> Acoustic calculation 3D, cylinder 40 dia long

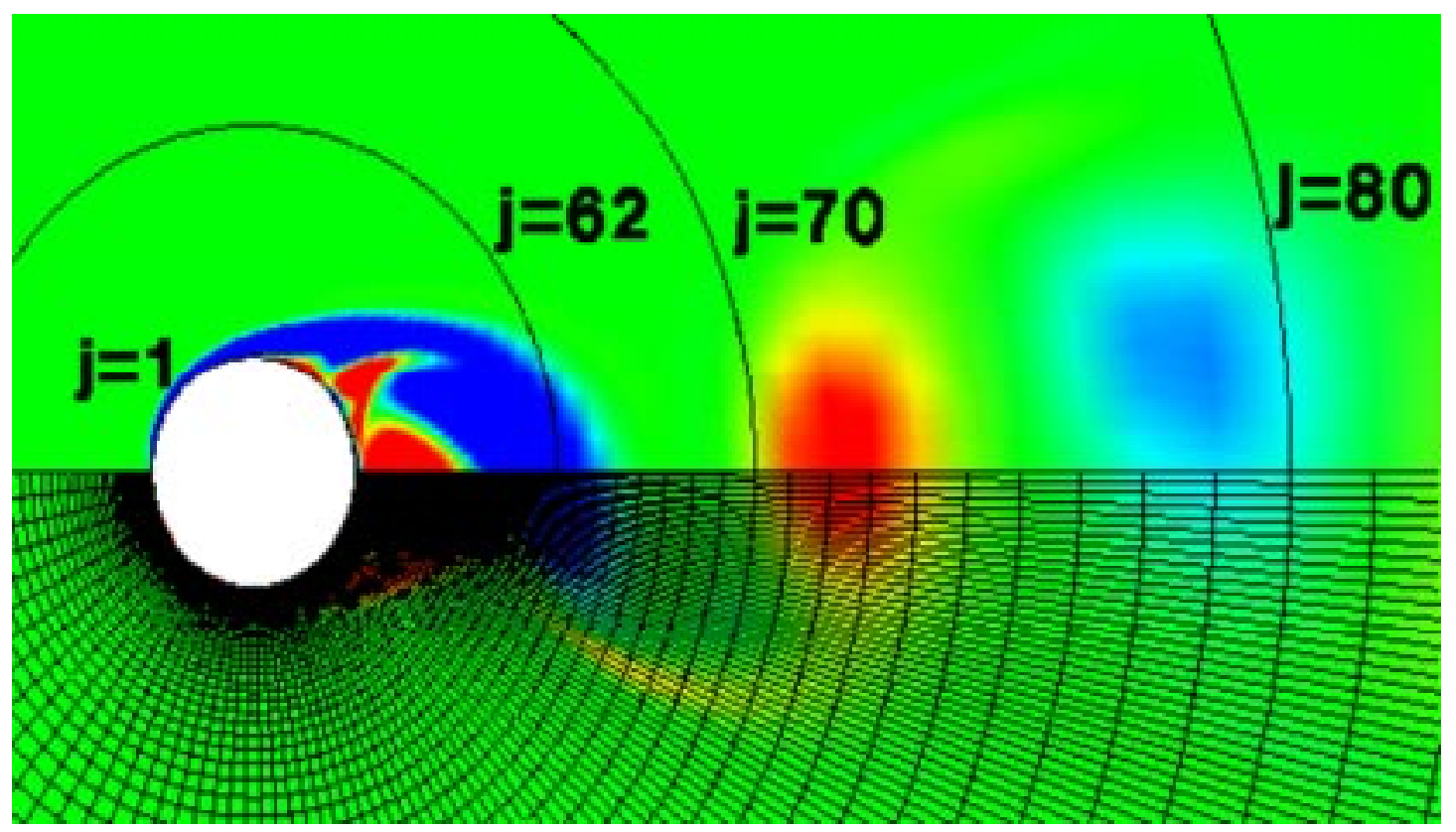

Vorticity field

from N-S computation

CFD grid 193x97

grid extends out 20 dia.

NASA Lanqlev Research Center. Hampton, VA 


\section{Noise Generated by Flow Over Cylinder}

- Location 128 dia from cylinder, $90 \mathrm{deg}$ from freestream
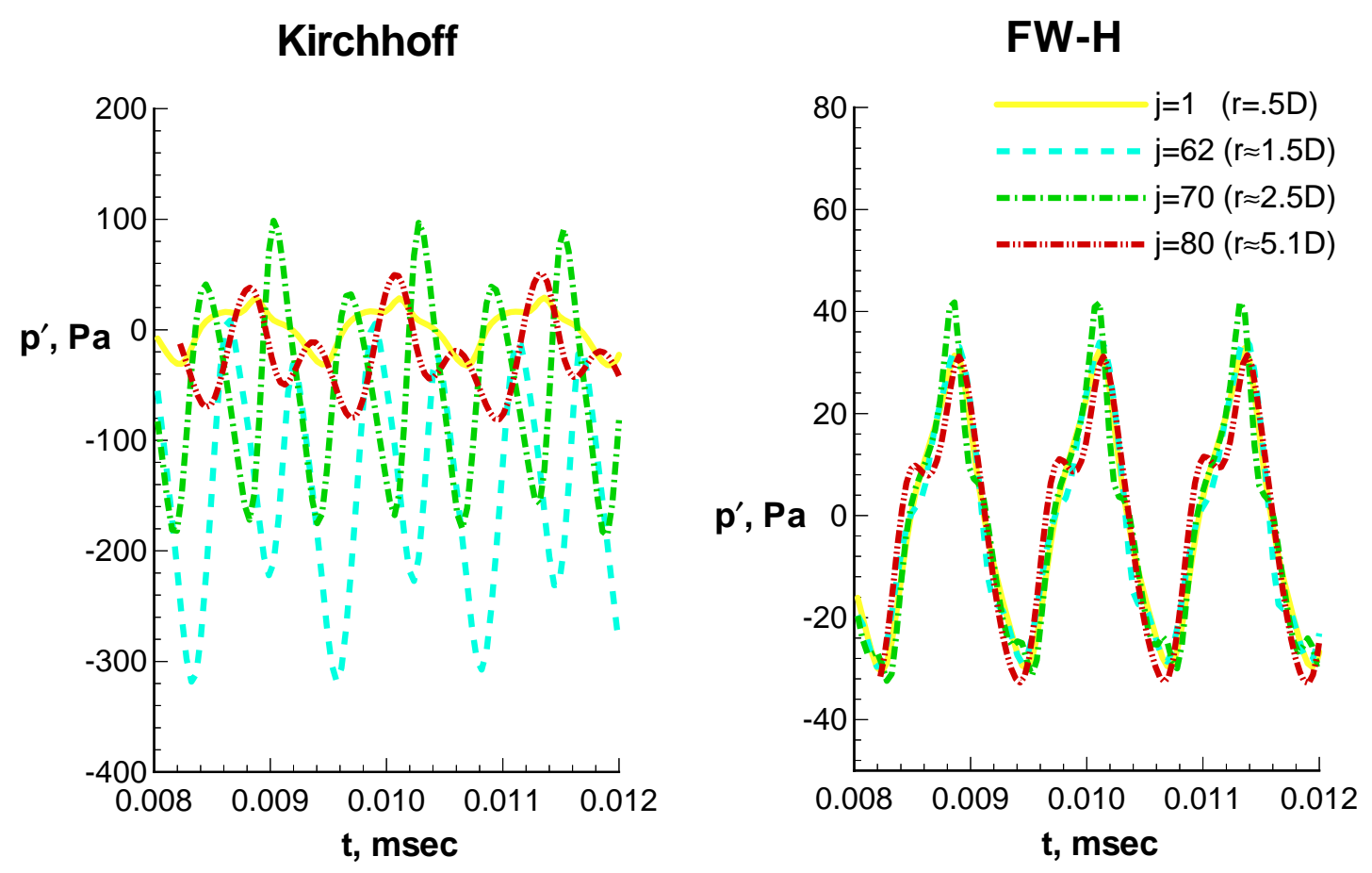

- FW-H predictions show small sensitivity to surface placement

- Kirchhoff predictions meaningless

NASA Lanqlev Research Center. Hampton. VA 


\section{Noise Generated by Flow Over Cylinder}

- Location 128 dia from cylinder, downstream
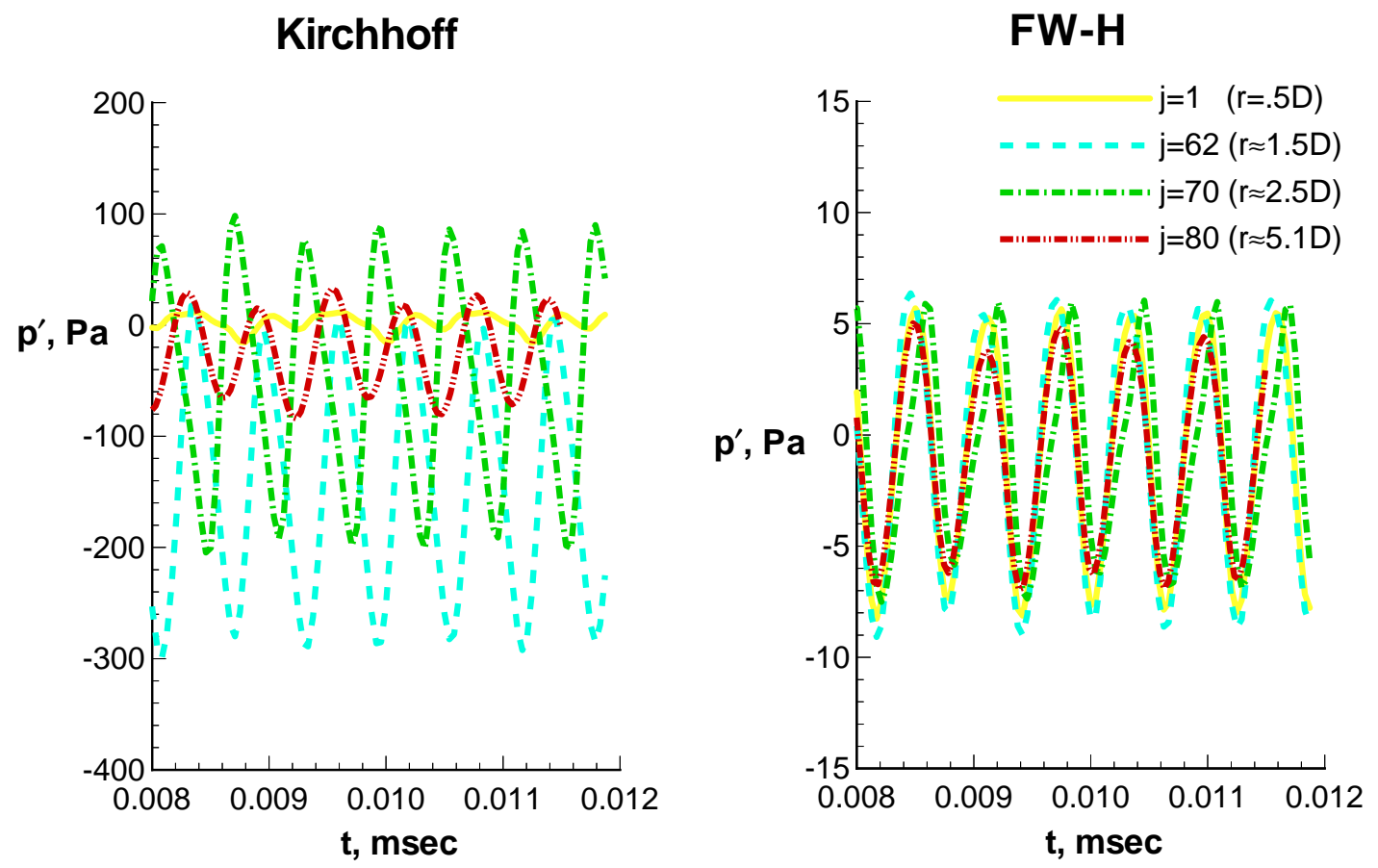

a differences in $\mathrm{FW}-\mathrm{H}$ prediction due to:

$>$ CFD inaccuracy $\quad>$ Increased integration error (grid size)

NASA Lanqlev Research Center. Hampton, VA 


\section{Conclusions}

- FW-H method of choice for aeroacoustic problems

$>$ conservation of mass and momentum built in

$>$ unified theory with thickness, loading, and quadrupole source terms

$>$ insensitive to integration surface placement

- FW-H approach the "better" than linear Kirchhoff because:

$>$ valid in linear and nonlinear flow regions

> surface terms include quadrupole contribution enclosed

$>$ physical noise components can be identified with two surfaces

- The Kirchhoff approach

$>$ valid only in the linear flow region (not known a priori)

- input data must satisfy the wave equation

- wakes and potential flow field can cause major problems

$>$ solution can be sensitive to placement of Kirchhoff surface

\section{NASA Lanqlev Research Center. Hampton. VA}

